\title{
Hydraulic losses in the spiral case of low specific speed pumps
}

\author{
Roman Klas ${ }^{1, a}$, František Pochylý ${ }^{1}$ and Pavel Rudolf ${ }^{1}$ \\ ${ }^{1}$ Brno University of Technology, Faculty of Mechanical Engineering, Victor Kaplan Department of Fluids Engineering, \\ Technická 2, 61669 Brno, Czech Republic
}

\begin{abstract}
This contribution is focused on analysis of pressure losses in spiral case of centrifugal pump with thick trailing edges and with recirculation channels. Recirculation channels have different geometrical configuration and influence the size of available specific energy as well as hydraulic efficiency. Subsequently, the contribution analyses the flow in spiral case itself with respect to its function and its filling with liquid. Studied phenomena affect the research of pumps with low specific speed, the stability of specific energy characteristic curves and also the configuration of recirculation channels.
\end{abstract}

\section{Introduction}

The characteristics of centrifugal pump are primarily by dependency of specific energy and efficiency on flow rate. These are static characteristics despite describing the actions that are considerably dependent on the rotation of the machine. However, also from these static curves can be deduced some dynamic characteristics of the machine e.g. stability of specific energy curve. As it is clear from definition of Euler turbine or pump equation the real specific energy curves will be influenced by the losses inside the centrifugal pump. The energy dissipation can therefore characterize stability of specific energy curve as well as dynamic characteristics of centrifugal pump. Therefore it is important how the impeller, gaps between hub or shroud and pump stator parts and spiral case participate on total pressure losses. It manifests that especially the spiral case can have a significant impact on the stability. The aim of this article will be therefore to assess the impact of losses in the spiral case on the operational characteristics of low specific speed pumps.

\section{The low specific speed pumps problems}

The main disadvantage of low specific speed pump design is low hydraulic efficiency. This fact is usually attributed to secondary flow in blade channels of impellers $[1,2]$. This flow features so called local eddy and causes problems with achieving the required values of specific energy in the operating point. Therefore new possibilities for the design of low specific speed pumps are searched. One of the possible solutions are the impellers with thick trailing edges and with recirculation channels. For their further development or optimization will be therefore important to know, how the losses in spiral case develop in comparison to the conventional impeller. From the view of losses different distribution of dissipated power between the spiral case and remaining parts also occurs [3].

\section{Impellers and CFD simulation}

Three centrifugal pumps with different impellers but common spiral body will be used to evaluate the dissipated power in the spiral case. Figure 1 offers the view of impellers with thick trailing edges with or without recirculation channels.
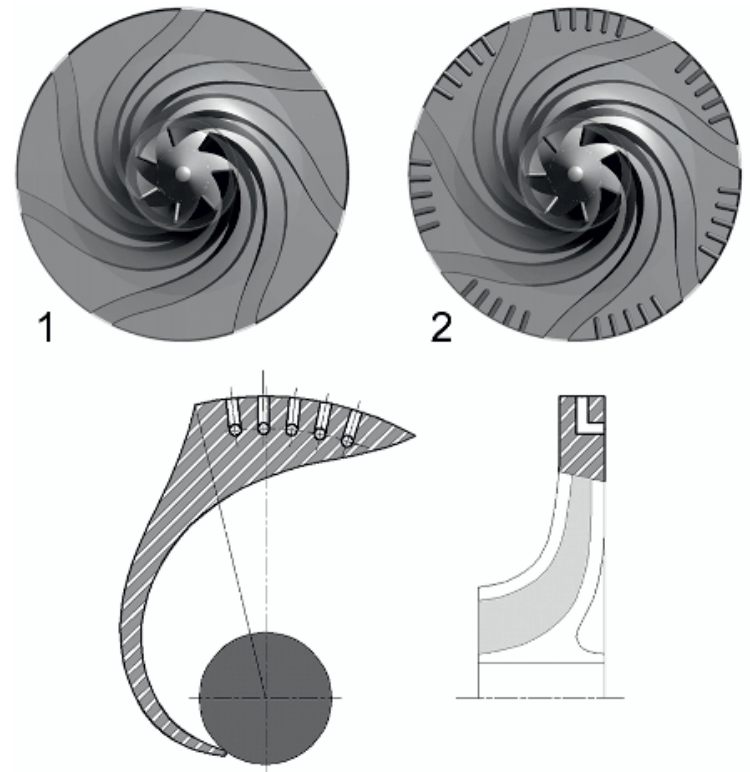

Fig. 1. Impellers with thick trailing edges.

\footnotetext{
${ }^{\mathrm{a}}$ Roman Klas: klas@fme.vutbr.cz
} 
The impellers are marked by number one and two. In the Table 1 are mentioned basic parameters of the impellers 1 and 2 .

Table 1. Basic parameters of the impellers 1 and 2.

\begin{tabular}{|c|c|}
\hline Number of blades & $\mathrm{z}=7$ \\
\hline Average value of blade angle $\beta_{2}$ & $\beta_{2}=63^{\circ}$ \\
\hline Impeller diameter $\mathrm{D}_{2}$ & $\mathrm{D}_{2}=0,314 \mathrm{~m}$ \\
\hline
\end{tabular}

Third impeller is conventional, but without the part of hub, Fig.2. The spiral case is the same for all three types of impellers.

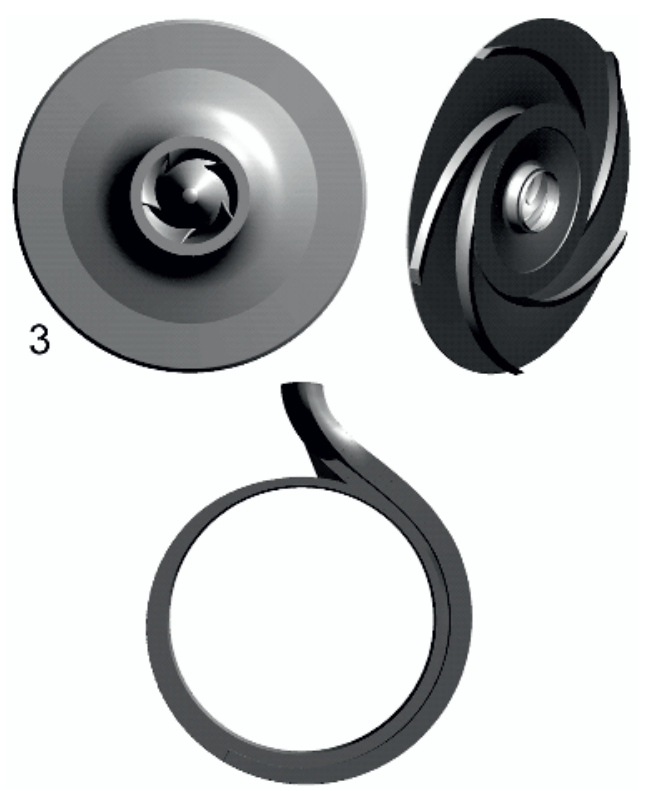

Fig. 2. Impeller No.3 and spiral case.

Basic parameters of conventional impeller are again mentioned in the Table 2.

Table 2. Basic parameters of the impeller No. 3.

\begin{tabular}{|c|c|}
\hline Number of blades & $\mathrm{z}=5$ \\
\hline Average value of blade angle $\beta_{2}$ & $\beta_{2}=24^{\circ}$ \\
\hline Impeller diameter $\mathrm{D}_{2}$ & $\mathrm{D}_{2}=0,314 \mathrm{~m}$ \\
\hline
\end{tabular}

The design parameters that are the same for all three pumps with impellers No. 1, No. 2 and No. 3 are mentioned in the Table 3.

Table 3. Common design parameters of pumps with impellers No. 1, No. 2 and No. 3.

\begin{tabular}{|c|c|}
\hline Flow rate & $\mathrm{Q}=0.00694 \mathrm{~m}^{3} \mathrm{~s}^{-1}$ \\
\hline Specific energy & $\mathrm{Y}=314 \mathrm{Jkg}^{-1}$ \\
\hline Rotational speed & $\mathrm{n}=24.167 \mathrm{~s}^{-1}$ \\
\hline Specific speed & $\mathrm{n}_{\mathrm{QE}}=\frac{\mathrm{nQ}^{0.5}}{\mathrm{Y}^{0.75}}=0.027$ \\
\hline
\end{tabular}

CFD simulations were used to analyse this problem. More detailed information about applied software and the settings is shown in the Table 4.

Table 4. Numerical simulation and calculation conditions.

\begin{tabular}{|c|c|}
\hline Preprocessor & GAMBIT 2.4.6 \\
\hline Solver & Fluent 12.1 \\
\hline $\begin{array}{c}\text { Number of computational } \\
\text { cells }\end{array}$ & $12-13$ millions \\
\hline Turbulence model & $\begin{array}{c}\text { realizable } \mathrm{k}-\varepsilon \\
\text { non equilibrium wall } \\
\text { function }\end{array}$ \\
\hline Calculation mode & $\begin{array}{c}\text { unsteady } \\
\text { sliding mesh } \\
\text { incompressible flow }\end{array}$ \\
\hline Boundary conditions & Inlet: velocity inlet \\
\cline { 2 - 3 } & Outlet: pressure outlet \\
\hline
\end{tabular}

\section{Main characteristics}

To ensure the relevance of CFD simulation results data are compared with experiment. First the specific energy characteristics and efficiency are introduced, Fig. 3 and Fig. 4.

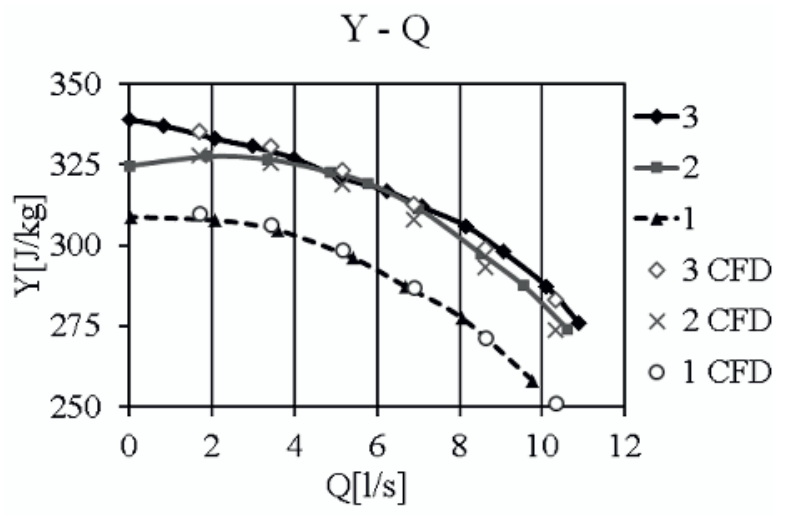

Fig. 3. Specific energy curves.

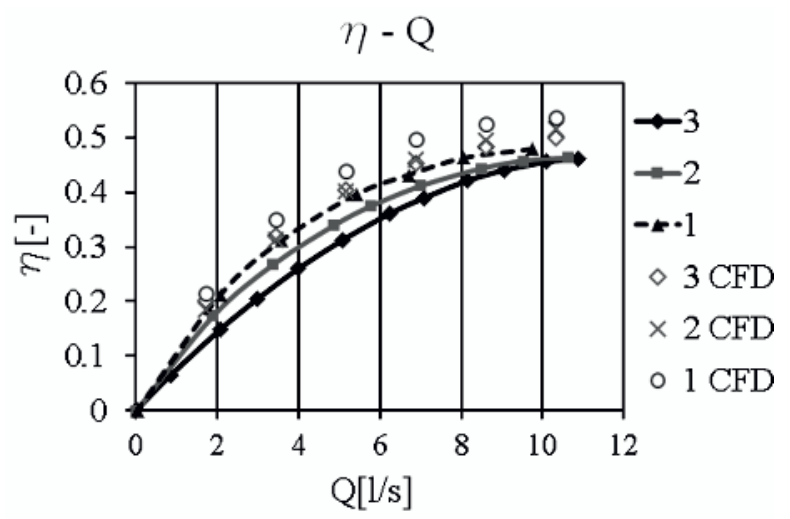

Fig. 4. Efficiency curves. 
In figure with specific energy curves (figure 3) can be noticed that curve No. 3 that corresponds to conventional impeller is stable. Specific energy curve No. 2 that corresponds to impeller with thick trailing edges and with recirculation channels is moderately instable around the shut-off point. And the curve No.1 that that corresponds to impeller with thick trailing edges without recirculation channels is on the border of stability around the shut-off point.

It is well known, that specific energy curve is stable [4] when following condition (1) is in the whole range of flow rates valid.

$$
\frac{\partial Y}{\partial Q}<0
$$

In the graph of efficiency (figure 4) some discrepancies are present, that are caused by the inability to determine the mechanical efficiency within numerical simulation. Efficiency is described by equation (2) and (3) for CFD.

$$
\begin{gathered}
\eta=\eta_{v} \eta_{h} \eta_{m} \\
\eta=\eta_{v} \eta_{h}
\end{gathered}
$$

Third most important characteristic will be total dissipated power. Differences from the measured values are again caused by the influence of mechanical efficiency. Differences are again caused by the influence of mechanical efficiency. Especially impellers with thick trailing edges, that were made from plastic, were bending due to axial forces during operation and rubbing against the spiral case.

To have an idea about the stability in the view of further analysis and evaluation, it will be necessary to introduce another criterion based on dissipated power free of mechanical and volume loss. The condition of stability [5] means, that for zero discharge $Q=0$ has to be

$$
\frac{\partial^{2} D_{H}}{\partial Q^{2}}>0 \wedge \frac{\partial D_{H}}{\partial Q}<0 .
$$

According to curves of dissipated power obtained from CFD simulation (for $Q=0$ ) results

$$
\begin{aligned}
& \left(\frac{\partial 2 D_{H}}{\partial Q} \leq 0\right)_{1 C F D}, \\
& \left(\frac{\partial 2 D_{H}}{\partial Q} \leq 0\right)_{2 C F D}, \\
& \left(\frac{\partial 2 D_{H}}{\partial Q}<0\right)_{3 C F D} .
\end{aligned}
$$

With respect to moderate instability of specific energy curves 2 CFD and 1 CFD (figure 3) and condition (4) these conditions (5) correspond to real properties of individual pumps.

\section{Energy dissipation}

In terms of evaluation of dissipation function the pump will be divided into several basic parts - spiral case, bladed channels, and gap between hub and pump stator parts and gap between shroud and pump stator parts. Standard definition of dissipation function was used to determine the magnitude of dissipated power (6).

$$
2 D_{H}=2 \eta \iiint_{V} v_{i j} v_{i j} d V
$$

Due to problems with the exact expression of the strain rate tensor $v_{i j}$ the balance of inputs and outputs (7) was used as well.

$$
2 D_{H}=\sum_{i=1}^{m} P_{\text {in } i}-\sum_{j=1}^{n} P_{\text {out } j}
$$

The error in expression of dissipated power by integration of dissipation function can be huge and results also vary according to applied calculation mode. For example they are different for steady and unsteady calculation, sliding mesh or frozen rotor.

Moreover, the spiral case was divided into smaller parts and dissipation function was evaluated in these smaller volumes (figure 5). In terms of circumferential angle the size of the element corresponds to five degrees.

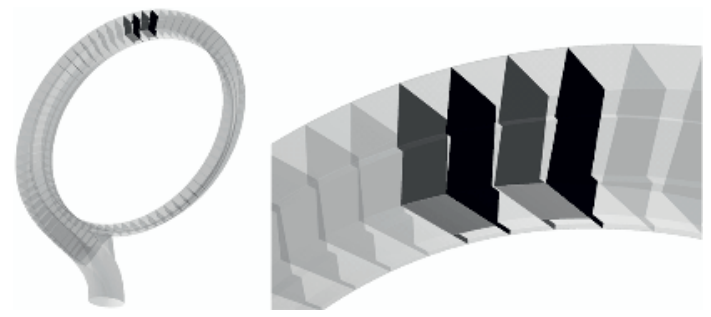

Fig. 5. Spiral case divided into smaller volumes.

\subsection{Energy dissipation within the pump}

First will be presented the overall characteristics for whole spiral case and blade channels. All results are valid for constant value of viscosity [6].

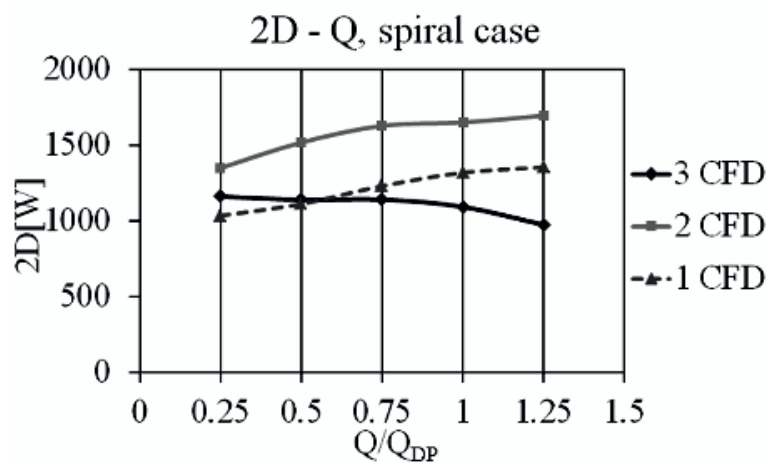

Fig. 6. Dissipated power in the spiral case.

It is obvious, that spiral case (figure 6) in impellers with thick trailing edges with and without recirculation 
channels contributes to instability of specific energy characteristic more significantly than in conventional impellers. This fact is evident from following equations (for $Q=0$ )

$$
\begin{aligned}
& \left(\frac{\partial 2 D_{H}}{\partial Q}>0\right)_{1 C F D}, \\
& \left(\frac{\partial 2 D_{H}}{\partial Q}>0\right)_{2 C F D}, \\
& \left(\frac{\partial 2 D_{H}}{\partial Q} \leq 0\right)_{3 C F D} .
\end{aligned}
$$

Design of the spiral case can therefore play more important role than for pump with conventional impellers in terms of shape and dimensions. The blade channels (figure 7) have significant stabilizing effect in conventional impeller.

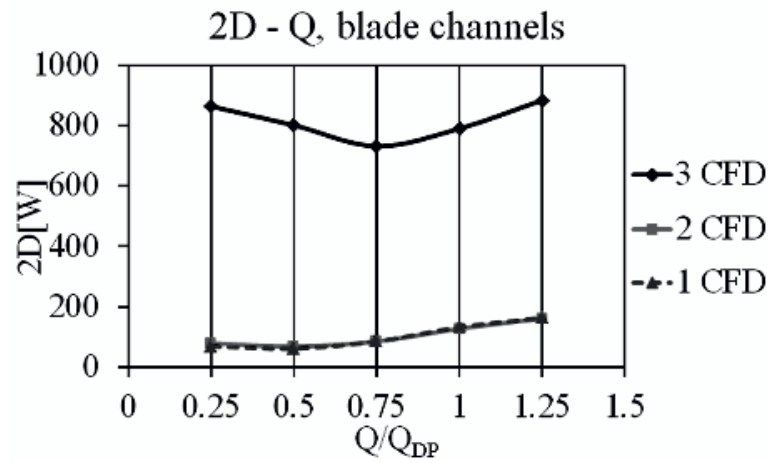

Fig. 7. Dissipated power in the blade channels.

The blade channels in impellers with thick trailing edges contribute to stability of specific energy curve only slightly (figure 7). See the following conditions

$$
\begin{aligned}
& \left(\frac{\partial 2 D_{H}}{\partial Q} \leq 0\right)_{1 C F D}, \\
& \left(\frac{\partial 2 D_{H}}{\partial Q} \leq 0\right)_{2 C F D}, \\
& \left(\frac{\partial 2 D_{H}}{\partial Q}<0\right)_{3 C F D} .
\end{aligned}
$$

For completeness are added the dissipated power curves in gaps between hub / shroud and pump stator parts. For pump with impellers with thick trailing edges can be seen certain similarity with the development of losses in the spiral case at low flows in terms of dissipated power in the space between the shroud and the pump casing (figure 8). This corresponds to a different character (mode) of flow in whole spiral case which is due to smaller outer area of blade channels. Another major factor in terms of losses will be different influence of the impeller around the spiral case nose [7].

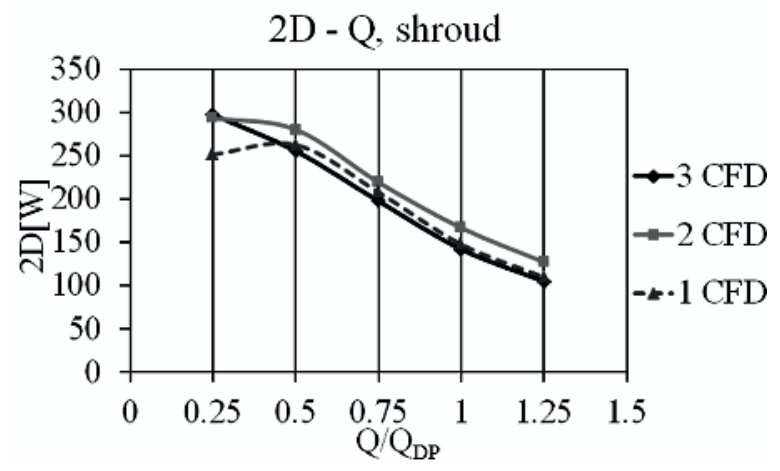

Fig. 8. Dissipated power in the gap between the shroud and the pump stator parts.

From the figure 9 it is evident, that the gap between the hub and pump stator parts has stabilizing effect in impellers with thick trailing edges. The hub for conventional impeller is not complete and the curve 3.1 corresponds to gap between blade channels and pump and the curve 3.2 to gap between the hub and pump.

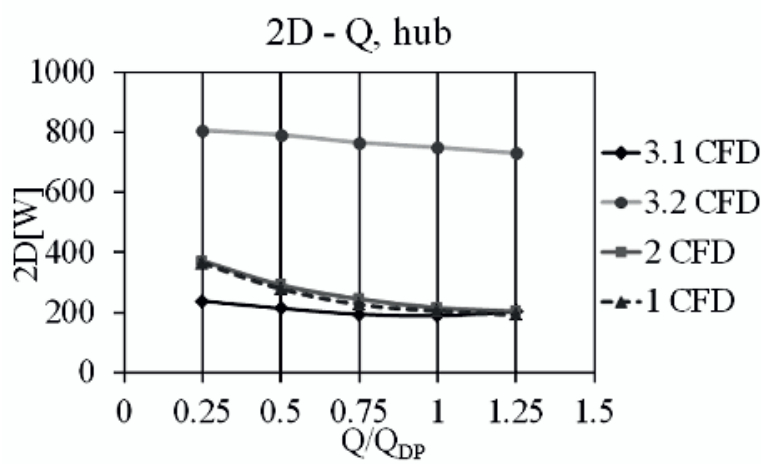

Fig. 9. Dissipated power in the gap between the hub and the pump stator parts.

\subsection{Energy dissipation within the spiral case}

If we look closer at losses in spiral case (figure 10), where $\varphi$ represents circumferential angle, it is evident, that the maximum of dissipated energy lies around the spiral nose, which is well known (angle 0 and 360). Near the spiral nose occurs even $25 \%$ of total losses in spiral case. Areas under the curve correspond to dissipated power. Individual values relate to the losses in small volumes, into which the spiral case was divided.

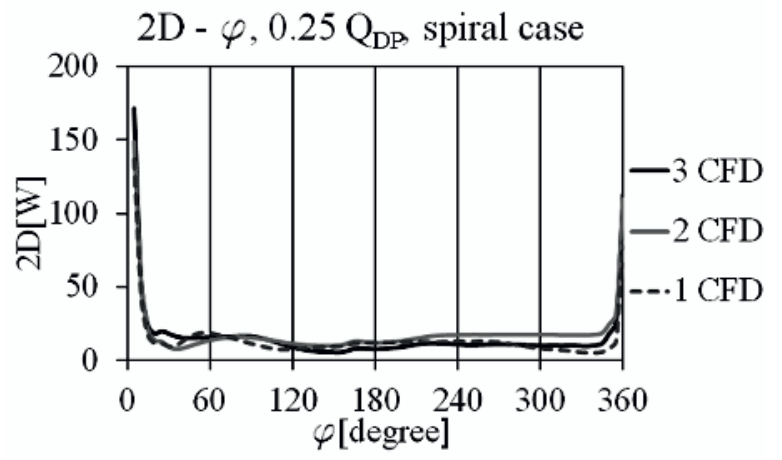

Fig. 10. Dissipated power in the spiral case. 
By using a different scale of vertical main axis, we can see near the shut-off point (figure 11) that after the flow stabilization downstream the spiral nose (angle 180 degree) the dissipation increases up to constant value. This value is highest for impellers with thick trailing edges with recirculation channels. Vice versa in impellers with thick trailing edges without recirculation channels the dissipation decreases upstream the spiral nose.

$2 \mathrm{D}-\varphi, 0.25 \mathrm{Q}_{\mathrm{DP}}$ spiral case

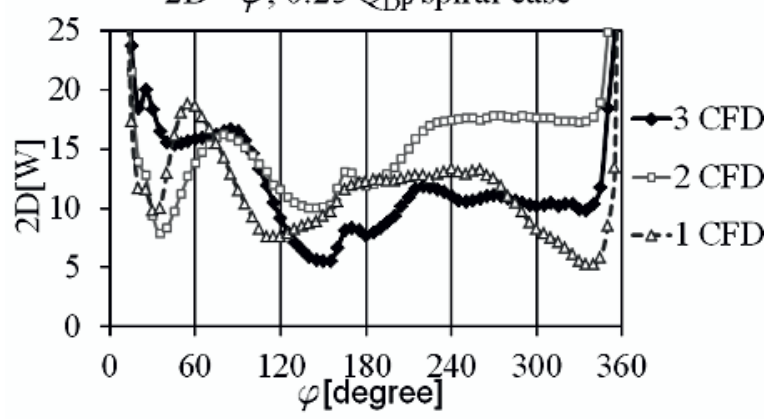

Fig. 11. Dissipated power in the spiral case, $0.25 \mathrm{Q}_{\mathrm{DP}}$.

From the figure 12 results, that the energy dissipation in great part of spiral case volume is lowest in conventional impeller. The peak (180 degree) in the both figures is enforced by the connection of individual parts of spiral case volumes.

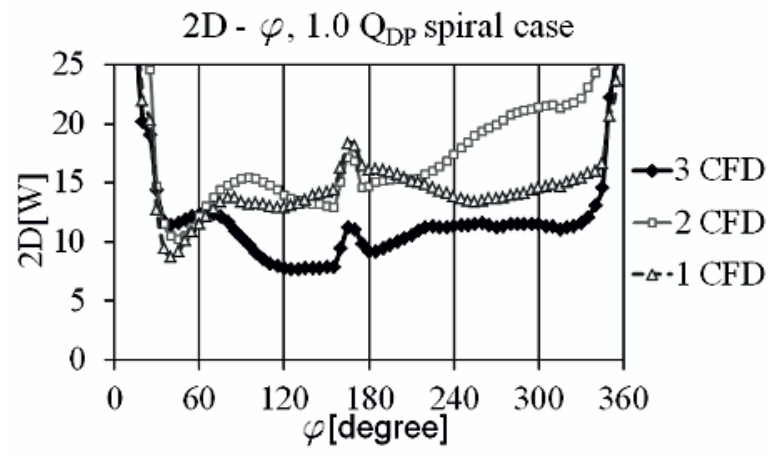

Fig. 12. Dissipated power in the spiral case, $1.0 \mathrm{Q}_{\mathrm{DP}}$.

It will be important, how the loss will develop around the spiral nose figure 13 and figure 14 , because the dissipated power reaches its maximum values there.

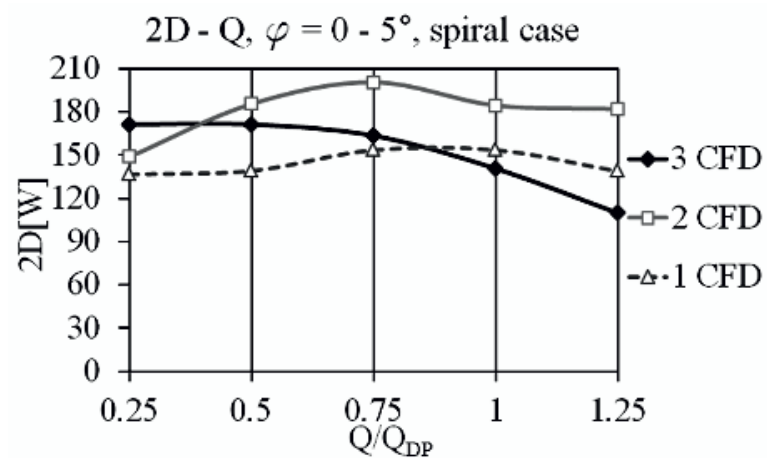

Fig. 13. Dissipated power in the part of spiral case, $\Delta \varphi 1$.

Around the shut-off point $0.25 \mathrm{Q}_{\mathrm{DP}}$ it seems the impellers with thick trailing edges to be the best. A significant thickness of trailing edges is in this case advantage. Vice versa around the design point it seems the variant No.3 to be optimal, which is related to more uniform filling of spiral case by liquid as well as to more uniform flow approaching spiral nose.

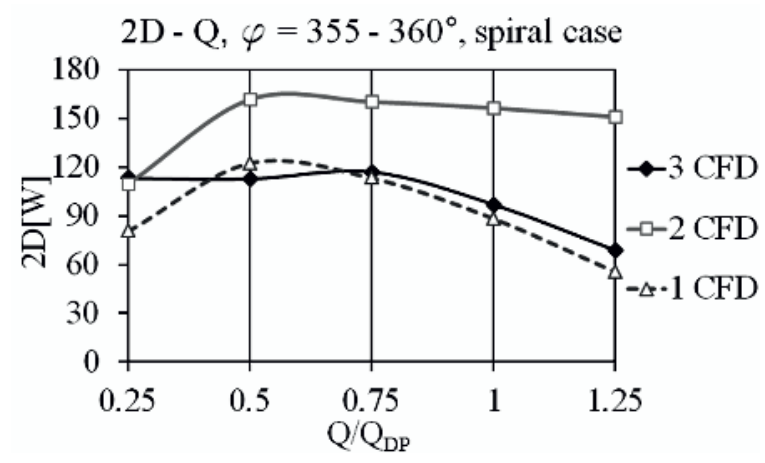

Fig. 14. Dissipated power in the part of spiral case, $\Delta \varphi 2$.

Figure 15 shows the ratio between the dissipated power in spiral case and the total dissipated power. For impellers with thick trailing edges is this value more than $60 \%$. The proper design of spiral case may therefore significantly affect the whole hydraulic layout as well as the final efficiency.

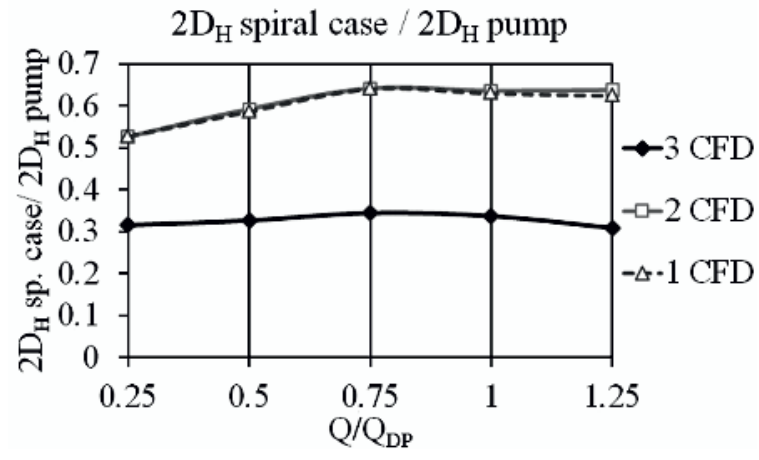

Fig. 15. Dissipated power ratio.

\section{Pressure pulsations}

Supplementary view point for analysis of the differences between the pumps with impeller with thick trailing edges and with conventional impeller will be the pressure pulsation in the spiral case. Angle $\varphi$ represents the spiral circumferential angle. The amplitude of static pressure pulsations is the smallest almost in the entire range for impeller without recirculation channels (figure 16).

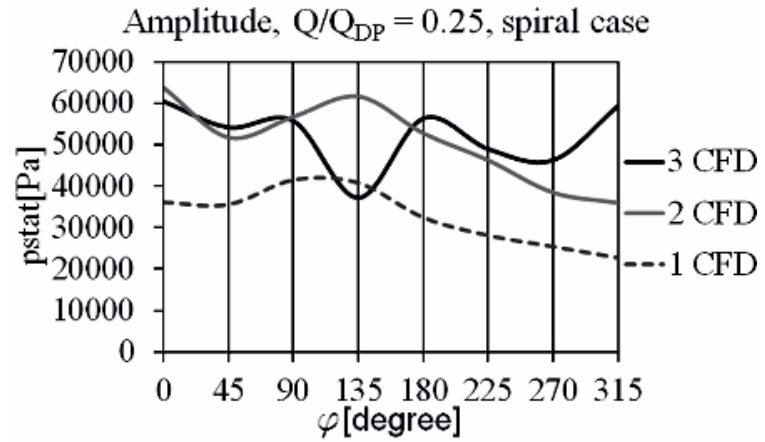

Fig. 16. Amplitude of static pressure, $Q / Q_{D P}=0.25$. 
In contrast, near the design point (figure 17) reaches the lowest amplitudes of pressure the impeller with the recirculation channels. So the recirculation channels decrease the static pressure pulsation in spiral case for higher flow rates.

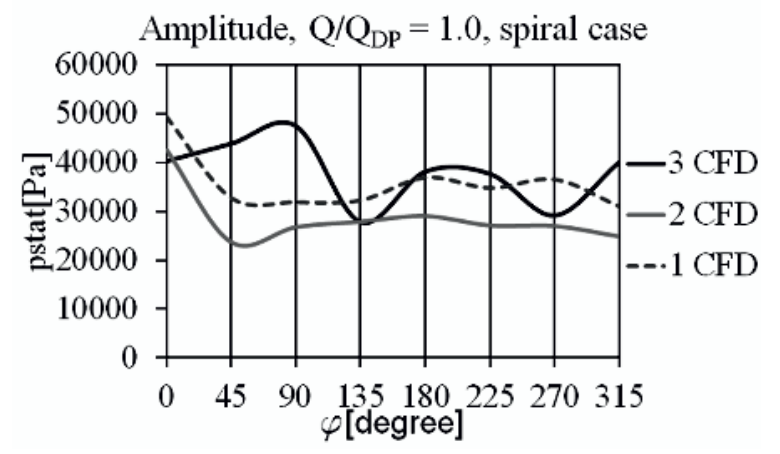

Fig. 17. Amplitude of static pressure, $Q / Q_{D P}=1.0$.

For completeness are added also the static pressure pulsations inside the pump close to the shut-off point figure 18 and in design point figure 19. Angle $\varphi$ represents rotation of the impeller.

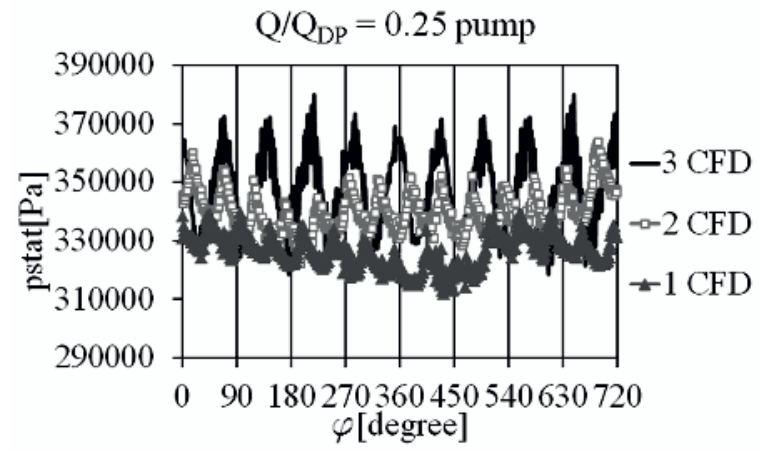

Fig. 18. Static pressure pulsations, $Q / Q_{D P}=0.25$.

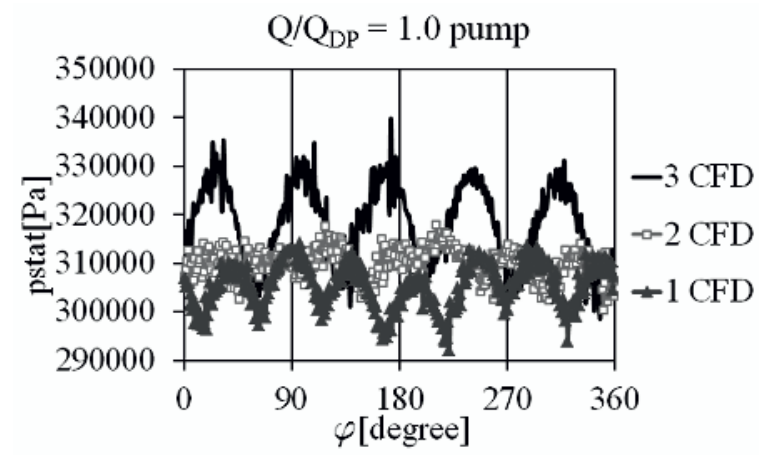

Fig. 19. Static pressure pulsations, $Q / Q_{D P}=1.0$.

Around the shut-off point and also near the design points are static pressure pulsations the highest for the conventional impeller. Also in this case the recirculation channels reduce the pulsations for the higher flow rates.

\section{Velocity field in the spiral case}

The field of absolute velocities is also one part of flow analysis in the spiral case. The effort will be especially aimed to point out the main differences of filling the spiral case by liquid. Only the examples of flow on which can be shown the basic differences will be selected. All following figures of velocity field in the spiral case will correspond to cuts with circumferential angle $\varphi$ equal to $270^{\circ}$ and for design flow rate. The flow is naturally depending on the position of impeller. Therefore the pair of figures for approximately corresponding position of blades to spiral nose $\alpha 1$ and $\alpha 2$ will be always presented. It is important to say, that the spiral case is designed as asymmetric for assembly reasons.

In figure 20 and figure 21 are shown absolute velocities in the cuts of spiral case for the pump with thick trailing edges and without recirculation channels.

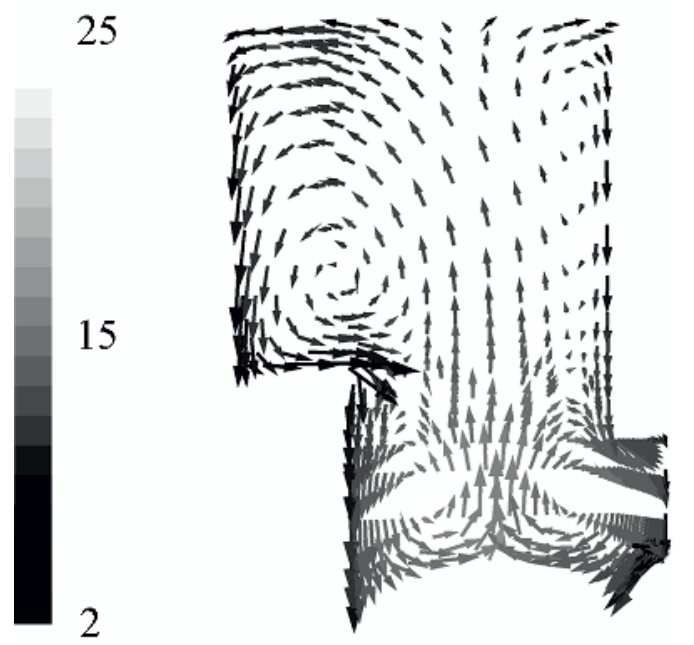

Fig. 20. Absolute velocities. Position of impeller No. 1 is $\alpha 1$.

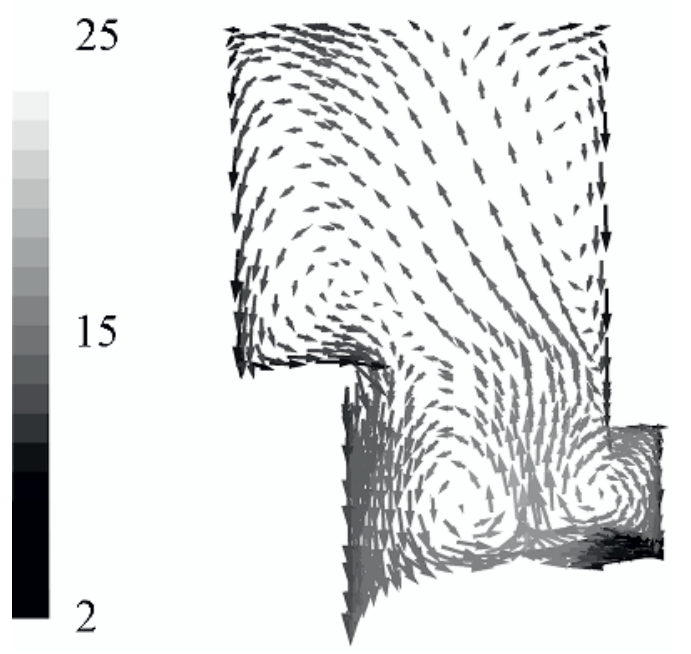

Fig. 21. Absolute velocities. Position of impeller No. 1 is $\alpha 2$.

The liquid enters the spiral case perpendicularly and two whirls (eddies) with opposite rotation arise. With change of the angle of impeller only the shift in location of both whirls happened. It is also obvious, that the liquid flow rate through the gap between shroud and pump stator parts (see below on the left side) is considerably higher than through the gap between hub and pump stator parts (see below on the right side).

The influence of recirculation channels can be well seen in figure 22 and figure 23 . The curve of velocities in 
the spiral case of pump with conventional impeller is displayed in figure 24 and figure 25.

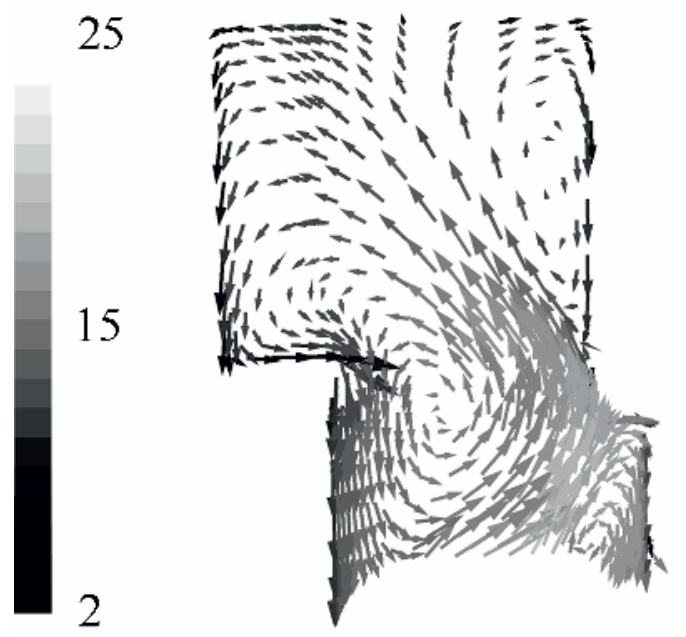

Fig. 22. Absolute velocities. Position of impeller No. 2 is $\alpha 1$.

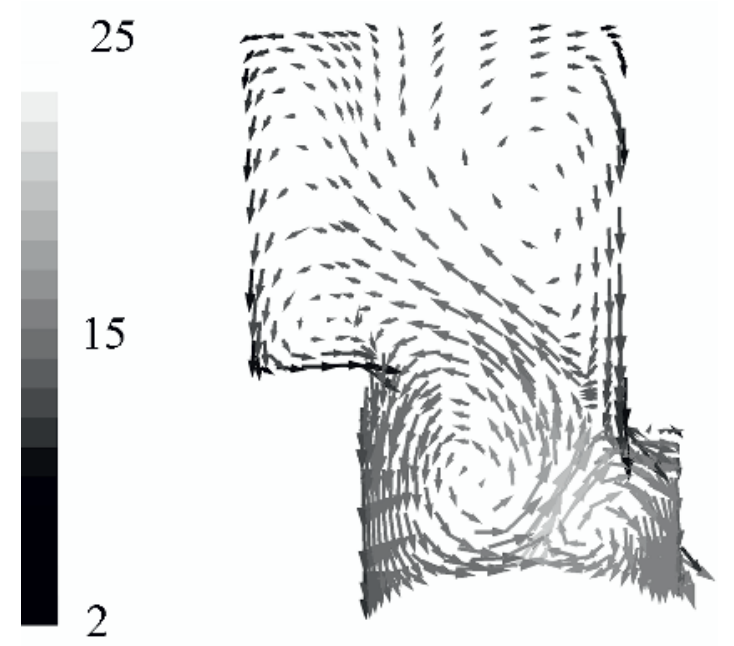

Fig. 23. Absolute velocities. Position of impeller No.2 is $\alpha 2$.

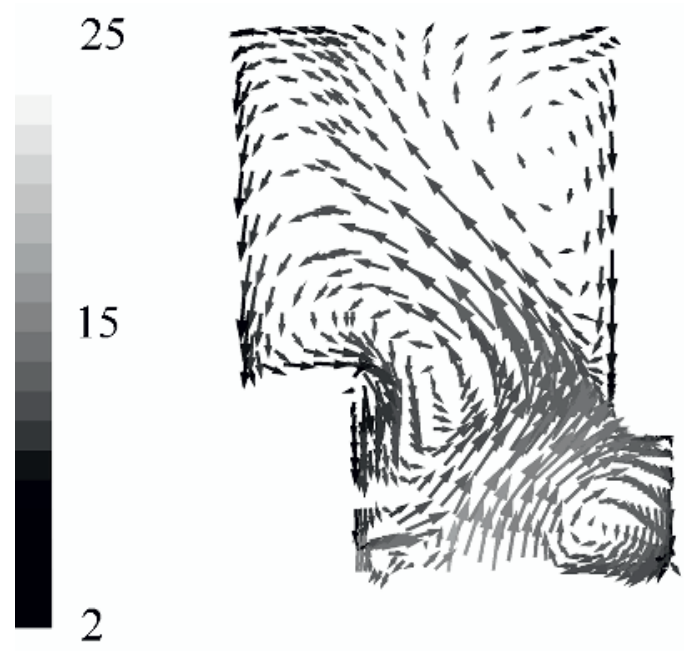

Fig. 24. Absolute velocities. Position of impeller No. 3 is $\alpha 1$.

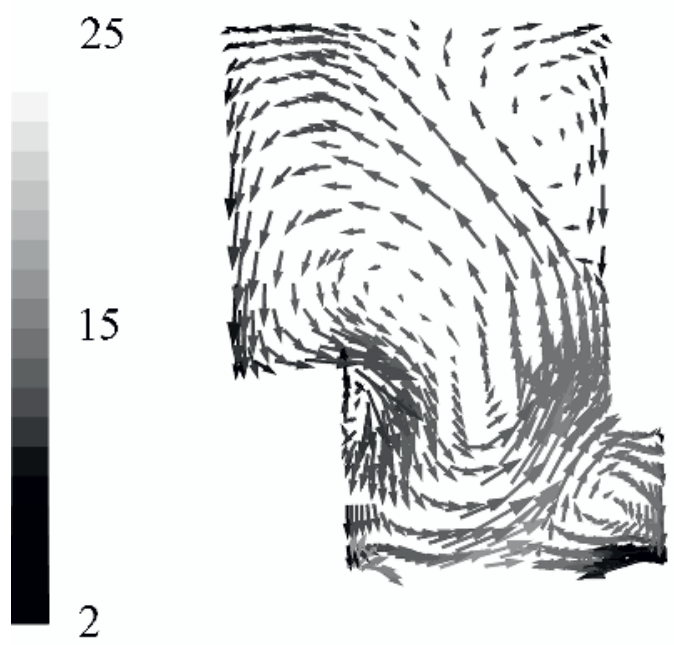

Fig. 25. Absolute velocities. Position of impeller No. 3 is $\alpha 2$.

In contrast to previous case in pump with impellers with recirculation channels (figure 22 a figure 23) significant deflection of liquid flow from perpendicular direction with respect to axis of pump rotation occurred. The size of whirls rising on impeller outlet is considerably different as well. The function of the recirculation channels is presented by the position of velocity vectors on the inlet to the gap between the hub and pump stator parts (see below on the right side).

Velocity field in the spiral case for pump with conventional impellers (figure 24 and figure 25) is mostly similar to the flow in pump with thick trailing edges and with recirculation channels. However, if we compare figure 22 and figure 24 it is obvious that the recirculation channels are reason of high velocities on the inlet to gap between the hub and pump stator parts (see below on the right side). The significant bending of liquid flow occurred as well. This all contributes to higher hydraulic losses, that can be observed in figure 12 for angle $\varphi$ equal to $270^{\circ}$. The lower velocities on outlet from conventional impeller are also important, which is understandable with respect to larger outlet width.

\section{Conclusions}

The specific energy curves for impellers with thick trailing edges may face to mild instability. The spiral case participates to eventual instability more in impellers with thick trailing edges than in conventional impellers.

In the view of losses around the spiral nose at lower flow rates the concept of impellers with thick trailing edges is preferable, dissipated power is lower or comparable. More uniform filling of spiral case by conventional impeller at higher flow rates however produces smaller hydraulic losses and the greater impact on the stability of specific energy curve as well.

The recirculation channels themselves in impellers with thick trailing edges cannot positively influence the stability of this curve and besides the increase of specific energy magnitude in whole range of characteristic participates also on increase of losses in spiral case. For higher flow rates are the losses in spiral cases for both 
types of pump with thick trailing edges higher than for conventional impellers. This brings a greater emphasis on selecting the appropriate shape of spiral case. Increasing flow rate also considerably decreases the amplitude of static pressure pulsation in spiral case for pump with recirculation channels. It can be lower than for pump with blade impellers and impellers with thick trailing edges without recirculation channels. The same conclusion can be drawn for pressure pulsations in whole pump as well.

In terms of impellers with thick trailing edges was then observed the stabilization effect of the gaps between the hub and pump stator parts.

From the curves of dissipated power can be observed the way, how individual parts of pump contribute to stability of specific energy characteristics as well as the magnitude, by which they participate to the total hydraulic pressure losses in the pump. Therefore it can be concluded, that a change of spiral case nose in pump with thick trailing edges can contribute to more acceptable dependence of dissipated power on flow rate. Mild extension of blade channels width in these pumps causes the increase of hydraulic losses in impeller by creation of secondary flow. Nevertheless, the wide blade channels have the stabilizing effect. The gap between the shroud and pump casing can also contribute to stability of specific energy curves by adjustment of the surface of spiral case and creating the labyrinths or grooves. However, these adjustments can be paid by decrease of the hydraulic efficiency.

In light of losses in spiral case in impellers with thick trailing edges and with recirculation channels the accent must be also put on shape of the spiral case. The significant curvature of liquid flow in inlet into the gap between hub and pump stator parts occurs due to recirculation channels. Therefore the narrowing of the spiral case in this place leads to increase of the hydraulic losses.

\section{References}

1. C. E. Brennen, Hydrodynamics of Pumps (New York: Oxford University Press, 1994)

2. J. F. Gülich, U. Bolleter, ASME J Vib Acoust, 114, 272-9 (1992)

3. K. Ch. Thin, M. M. Khaing, K. M. Aye, Engineering and Technology, 36, $422-429$ (2008)

4. J. F. Gülich, Centrifugal Pumps (Springer - Verlag, Berlin, 2008)

5. F. Pochylý, M. Haluza, S. Drábková, Engineering Mechanics 2009 - book of extended abstract, 208215 (2009)

6. W. G. Li, International Journal of Heat and Fluid Flow, 21, 207 - 212 (2000)

7. Y. W. Wong, W. K. Chan, W. Hu, Artificial Organs, 31(8), 639-645 (2007)

\section{Acknowledgement}

Project CZ.1.07/2.3.00/30.0005 "Podpora tvorby excelentních týmů mezioborového výzkumu na VUT" is gratefully acknowledged for support of this research. 